\title{
Targeting properdin in the treatment of atypical haemolytic uraemic syndrome: better than eculizumab?
}

\author{
Kate Smith-Jackson, Kevin J. Marchbank \\ NRCTC and Newcastle University, The medical school, Farmington place, Newcastle-upon-Tyne, UK \\ Correspondence to: Kevin J. Marchbank, PhD. Institute of Cellular Medicine, NRCTC, Newcastle University and Newcastle-upon-Tyne Hospitals \\ Trust, 3rd floor William Leech Building, The Medical School, Farmington Place, Newcastle upon Tyne, NE2 4HH, UK. \\ Email: kevin.marchbank@newcastle.ac.uk. \\ Provenance: This is an invited Editorial commissioned by Section Editor Linpei Jia, MD, PhD (Department of Nephrology, Xuanwu Hospital of \\ Capital Medical University, Beijing, China). \\ Comment on: Ueda Y, Miwa T, Gullipalli D, et al. Blocking Properdin Prevents Complement-Mediated Hemolytic Uremic Syndrome and Systemic \\ Thrombophilia. J Am Soc Nephrol 2018;29:1928-37.
}

Submitted Sep 30, 2018. Accepted for publication Oct 11, 2018.

doi: 10.21037/atm.2018.10.35

View this article at: http://dx.doi.org/10.21037/atm.2018.10.35

The need for better anti-complement drugs in certain complement mediated diseases and disease states remains pressing (1). The recent data published by Ueda et al. (2), appears to offer another highly effective solution to target complement activation in atypical haemolytic uraemic syndrome (aHUS). AHUS is a primary thrombotic microangiopathy (TMA), clinically characterized by acute kidney injury, microangiopathic haemolytic anaemia, and thrombocytopenia (1). AHUS, along with paroxysmal nocturnal haemoglobinuria (PNH) and $\mathrm{C} 3$ glomerulopathy (C3G), are archetypal complement mediated diseases. With aHUS and PNH being treated, on the whole, through use of the C5 functional blocking antibody therapy eculizumab $\left(\right.$ Soliris $\left.^{\mathrm{TM}}\right)(1,3)$. Despite the clear role of complement in C3G, its successful treatment using eculizumab has had mixed success (1).

In the most comprehensive analysis to date, $>600$ rare variants in 10 complement genes have been identified in over 3,000 aHUS patients (identified in 6 reference centres including our own) (4), the majority of these changes are found in the alternative pathway of complement activation (Figure 1), particularly its regulators, factor $\mathrm{H}(\mathrm{FH})$ and membrane cofactor protein (MCP, CD46). Indeed, the first described mouse model of aHUS used a genetic deletion of the last 5 SCRs of FH, i.e., FH delta CCP16-20, (a change not seen in man), to demonstrate that the carboxy terminus of FH is essential to prevent aHUS-like disease (5) and confirmed that surface localisation of $\mathrm{FH}$ is critical to prevent aHUS. The relative success of that model, with respect to replication of the pathological features of the human disease in mouse has led to several attempts to generate even more realistic models of aHUS based on single amino acid variations. One such model is the FH W1206R or $\mathrm{FH}^{\mathrm{R} / \mathrm{R}}$ model (6). It is based on a $\mathrm{C}$ terminal $\mathrm{FH}$ mutation found in aHUS patients that in homozygous mice, allows uncontrolled $\mathrm{C} 3 \mathrm{~b}$ deposition on host tissue leading to renal failure, stroke and retinopathy $(2,6)$. The resulting model appears to represent an aggressive TMA with occlusive microvascular and macrovascular disease. It is worth noting that only a $5^{\text {th }}$ of aHUS patients display extrarenal complications while retinal involvement and stoke are rare events in aHUS patients (7). In the $\mathrm{FH}^{\mathrm{R} / \mathrm{R}}$ model, approaching one third of the tested mice displayed symptoms of stroke and ischemic retinopathy. The onset of organ thrombosis and renal injury occurred within the first week of life, with sudden death being observed on numerous occasions, culminating in just under half of the $\mathrm{FH}^{\mathrm{R} / \mathrm{R}}$ animals (but none of the heterozygous variant animals) being removed from the study by 30 weeks post-partum (6). Arguably, this provides an ideal and stringent test for therapeutics destined to reverse the effects of rampant uncontrolled complement activation at the cell surface. Furthermore, as mice develop disease from 3-4 weeks of age, with $\mathrm{FH}^{\mathrm{R} / \mathrm{R}}$ mice being smaller and thinner than $\mathrm{FH}^{\mathrm{R} /}$ 


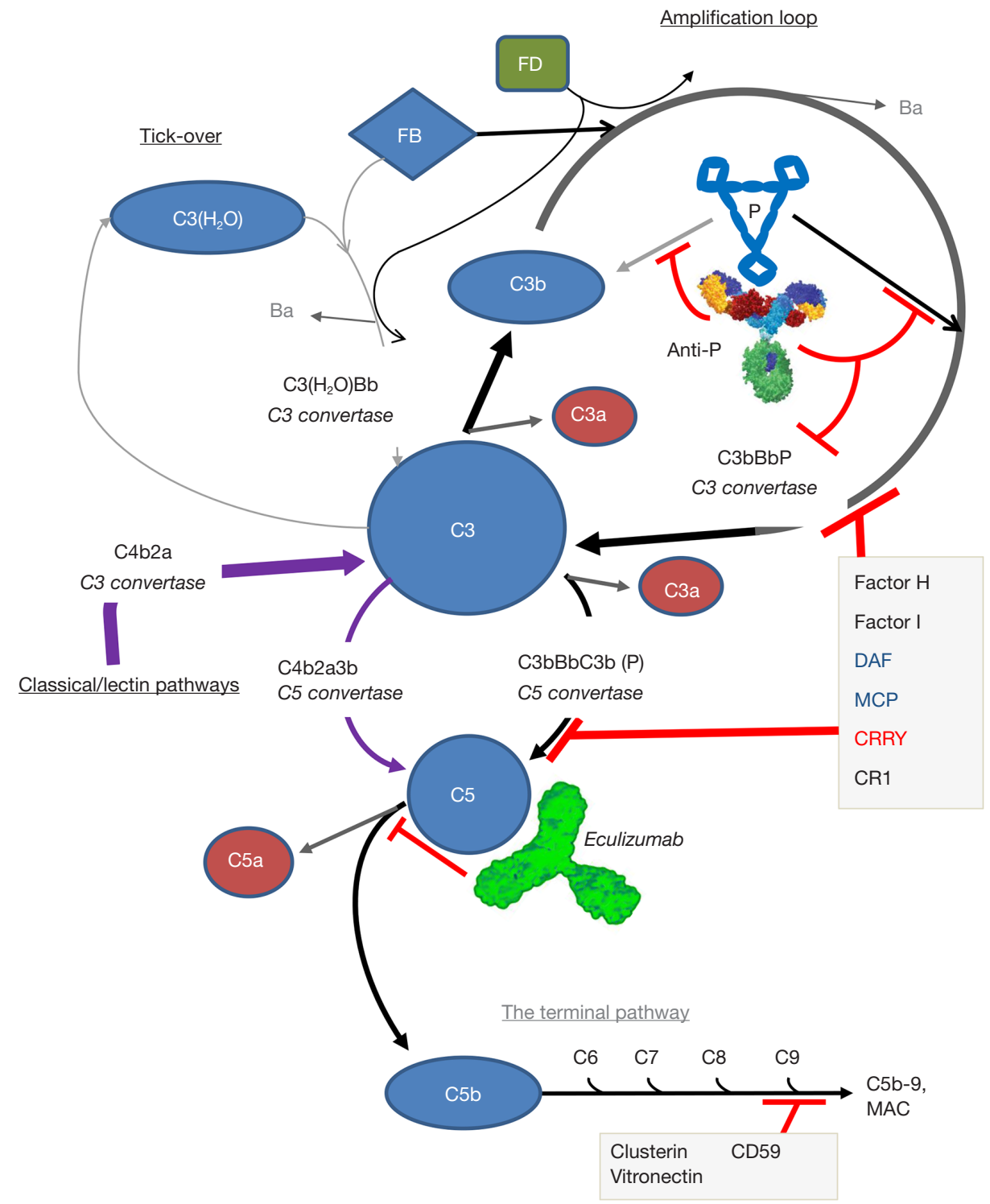

Figure 1 An AP focused overview of the complement system. Activation of complement (C) can occur via three pathways-the classical, lectin, and AP, depending on the trigger, with all resulting in the cleavage of the pivotal molecule, $\mathrm{C} 3$ into $\mathrm{C} 3 \mathrm{a}$ and $\mathrm{C} 3 \mathrm{~b}$ by $\mathrm{C} 3 \mathrm{convertases}$. The AP is perpetually and slowly active through a mechanism known as tick-over i.e., the spontaneous hydrolysis of $\mathrm{C} 3$. This $\mathrm{C} 3\left(\mathrm{H}_{2} \mathrm{O}\right)$ species can form an initial fluid phase $\mathrm{C} 3$ convertase upon association with $\mathrm{FB}$ that is cleaved by the proteolytic enzyme $\mathrm{FD}$, generating $\mathrm{C} 3\left(\mathrm{H}_{2} \mathrm{O}\right) \mathrm{Bb}$ and $\mathrm{Ba}$. $\mathrm{C} 3\left(\mathrm{H}_{2} \mathrm{O}\right) \mathrm{Bb}$ can now interact with $\mathrm{C} 3$ to generate $\mathrm{C} 3 \mathrm{~b}$ and $\mathrm{C} 3 \mathrm{a}$ within the amplification loop. Bound $\mathrm{C} 3 \mathrm{~b}$ on surfaces forms a platform for $\mathrm{FB}$ binding, creating $\mathrm{C} 3 \mathrm{bB}$, which is rapidly cleaved by $\mathrm{FD}$ to $\mathrm{C} 3 \mathrm{bBb}$ (a new $\mathrm{C} 3$ convertase) which can readily bind properdin $(\mathrm{P})$, which stabilises the newly formed convertase $(\mathrm{C} 3 \mathrm{bBbP})$. The $\mathrm{AP}$ can be initiated by properdin as this molecule recognizes target surfaces (such as apoptotic bodies, cells) and subsequently recruits $\mathrm{C} 3 \mathrm{~b}$ and $\mathrm{FB}$ to form stabilized C3 convertases. As the classical and lectin pathway also generate $\mathrm{C} 3 \mathrm{~b}$, the amplification loop within the AP is incredibly important to propagate the activity of complement per se. As sufficient saturation is reached $\mathrm{C} 3 \mathrm{~b}$ can attach to preformed $\mathrm{C} 3$ convertase complexes to form $\mathrm{C} 5$ convertases that allow the terminal pathway of complement to proceed i.e. C5 is cleaved into C5a and C5b. C5b then recruits C6, C7, C8, and up to thirteen C9 molecules to form the membrane attack complex (MAC; C5b-9) which can punch holes in cell membranes. Naturally, all this 'fire power' needs control and so many regulators of complement exist (selection noted in grey boxes) with Factor $\mathrm{H}$ and Factor I being critical for control of the AP. The site of action of eculizumab and anti-properdin are also illustrated. AP, alternative pathway; FB, factor B; FD, factor D. 
${ }^{\mathrm{W}}$ or $\mathrm{FH}^{\mathrm{W} / \mathrm{W}}$ littermates, this is a relatively cost-effective model to run compared to the $\mathrm{FH}$ knockout model of $\mathrm{C} 3 \mathrm{G}$ (for instance).

Thus, the clear success of anti-properdin therapy by Udea et al. (2), with complete blockade of properdin function and prevention of sudden death and reduction of disease markers over the 2 months analysed in the $\mathrm{FH}^{\mathrm{R} / \mathrm{R}}$ mouse is a significant finding. These findings clearly echoed results from the backcross of the $\mathrm{FH}^{\mathrm{R} / \mathrm{R}}$ mice with properdin knockout $\left(\mathrm{P}^{-/-} ; \mathrm{P}^{\text {flox/flox }}\right.$ crossed with EIIa-Cre) mice carried out in the same study which demonstrated a highly important role of properdin in fostering the complement activation that drives the pathological outcomes of TMA. This is logical based on the central role that properdin plays in cross-talk between platelets and neutrophils, driving another important feedback loop of activation of each cell type which drives thrombo-inflammation (8).

The AP is a uniquely important part of the complement system (9) and interacts with the other activation pathways to amplify the innate and acquired immune response (10). Indeed, too many, it is this amplification or feedback loop potential that defines the AP. For instance, the AP has been shown to account for approximately $80 \%$ of terminal pathway activation when complement activity is initiated by either the classical or lectin pathways. Indeed, properdin inhibitors have been suggested to be approximately four fold more effective than C5 inhibitors in preventing sheep red blood cell lysis in the presence of $\mathrm{C}$ terminal $\mathrm{FH}$ perturbation (8). Thus, the targeting of properdin, an important positive regulator of the AP (8) (see Figure 1), has several obvious/attractive points. Primarily properdin is essential for effective AP function although some AP function can occur in the absence of properdin (11) and will leave the Classical and Lectin activation pathways intact. Properdin is produced mainly by leucocyte populations (as well as adipocytes and stimulated endothelial cells) and is found in the circulation at relatively low concentrations of 4-25 $\mu \mathrm{g} / \mathrm{mL}$ (about 5 fold less than C5, at $75 \mu \mathrm{g} / \mathrm{mL}$ ) (12), so one of the potential advantages that anti-properdin treatment may offer compared to eculizumab is the need for a lower dose of the antibody to deplete/block the protein. However, that remains to be seen based on the data from the mouse model, i.e., platelet levels and haemoglobin were only fully stabilised when the $2 \mathrm{mg}$ per mouse per week dose (roughly between $150-100 \mathrm{mg} / \mathrm{Kg}$ across the study) was used. This is a markedly higher dose than that currently used for eculizumab, at any phase of treatment (3) or 3 fold the effective dose for use of BB5.1, a murine ortholog of eculizumab (13). While C5 expression, which is produced predominately in the liver, can increase in response to inflammation, arguably local properdin levels can and do rise more rapidly in response to inflammatory stimuli and, in the case of endothelial cells, laminar shear stress (12). The conditions that provoke TMA are likely to provoke a rapid increased in local properdin expression levels (8). This may explain the need for use of high dose of anti-properdin to completely block properdin function in the $\mathrm{FH}^{\mathrm{R} / \mathrm{R}}$ model. Similar doses were used in other studies using different anti-properdin blocking antibodies to prevent complement mediated disease (14) not initiated by AP activation. In short, these murine models do suggest the need for a high dosing strategy to maintain properdin deficiency.

Of course, we at the National Renal Complement Therapeutics Centre (NRCTC) of the United Kingdom were one of the first to recognise that eculizumab (Soliris ${ }^{\mathrm{TM}}$ ) through the complete blockade of the terminal pathway of complement (Figure 1) is a highly effective treatment in many patients with aHUS (15). However, we also accept that eculizumab is not a panacea (1) in this disease and has had mixed success in the treatment of $\mathrm{C} 3 \mathrm{G}$. Furthermore, due to the high cost of eculizumab, plasma exchange remains the only affordable treatment in many parts of the world. One of the major risk factors with the use of any anti-complement drug is the increased risk of infection. Use of eculizumab essentially completely switches off two important components of our immune system, i.e., generation of membrane attack complex and C5 a naphylatoxin. The loss of these effector mechanisms leaves patients treated with the drug highly susceptible to infections that can cause meningitis, requiring prior vaccination and, in certain treatment centres including the UK, prophylactic antibiotics before treatment can proceed $(1,3)$. So would properdin depletion be any safer? This is arguable, extrapolating from the role of properdin in promoting the amplification loop of the AP and how that translates into terminal pathway activation (8), its perhaps not surprising that properdin deficiency is associated with a markedly increased (250 fold) risk of meningococcal infection, particularly to serotypes such as W-135 and Y (16) compared with complement sufficient individuals. Furthermore, properdin deficiency is estimated to increase the rate of mortality during infection to $33 \%$ in comparison with rates of $5-7 \%$ for individuals with terminal complement component deficiencies or are complementsufficient (16). Studies in animal models with properdin deficiency also highlight that properdin is crucial for 
robust host defence against certain, but not all, microbial pathogens (17). This is logical in that loss of properdin will blunt activation of the terminal pathway regardless of the initiation pathway (14) as well as interfere with C3a/ C5a anaphylatoxin signalling and opsonisation which is important to both innate and adaptive cell activation/ responses. This deficit is balanced by the fact that antiproperdin therapy, similar to other approaches targeting the AP $(8,18)$, will allow a certain level of classical and lectin pathway mediated terminal pathway/anaphylatoxin activity to remain intact. Of course, it's notable that concerns surrounding increased infectious risk have not prevented an anti-properdin therapy, CLG561, from reaching phase II clinical trials in the treatment of age-related macular degeneration (19). Overall, these data suggest that total blockade of properdin function (if achievable systemically) in the patient population will require considerations surrounding increased risk of infection that are broadly similar to C5 blockade.

A further note of caution on this topic is that while preventative vaccination against meningitis is routine in the use of anti-complement drugs, it is important to also consider in patients with deregulated complement systems that an inefficient/or perturbed complement system may have significant detrimental effects on vaccine responses (10) i.e., protective vaccination in patients with rare variants in complement proteins may be significantly compromised and therefore less likely to provide the protection that would otherwise be expected. That sentiment is upheld by current patient data, i.e., patients on eculizumab still remain at high risk of meningococcal disease despite vaccination (20). However, there is evidence that vaccination against meningococcus can be effective in properdin deficient patients (16) and prophylactic use of penicillin can further reduce this risk (1).

Over the last decade, we have successfully generated several unique recombinant mouse $\mathrm{C} 3$ molecules based on variants found in human $\mathrm{C} 3$ that associated with aHUS or $\mathrm{C} 3 \mathrm{G}$, with an aim to better understand how certain pathological variants in $\mathrm{C} 3$ associate with particular disease pathology. In doing so, we demonstrated that we could transfer functional changes from patients with aHUS to a mouse model (21). Mice with a single point mutation in C3, i.e., pC3.D1115N (gain-of-function, C3 GOF) on both gene copies of $\mathrm{C} 3$ rapidly develop aHUS with a pathology that is identical to the original patients described with the variant. Furthermore, our data also confirms that C5 deletion or blockage (using BB5.1) rescues mice from the disease phenotype (22) and therefore validates the model for testing of other anti-complement drugs (SmithJackson et al., accepted subject to revision, JCI). However, in preliminary analysis using $\mathrm{H} 4$ (14), a properdin function blocking antibody, we saw little if any positive effects of using the treatment (Smith-Jackson et al., unpublished). While many reasons for this poor response in our aHUS model exist at this present time, it does potentially remind us that all pathological mechanisms in aHUS are not the same. Indeed, C3 variants may produce disease pathology which sits between aHUS and C3G. This has important implications as deletion of properdin in the $\mathrm{FH}$ knockout mouse (model of C3G) significantly exacerbated disease (23) as measured at 38 weeks. This was similar, if not, much more marked, in another model where mice succumbed to disease between 8-12 weeks in the presence of low levels of a mutant FH and FP deficiency compared to 12-month disease onset when just low FH was present (24). The reason for properdin deficiency not being protective in models of C3G remains largely uncertain as several studies demonstrated that deletion or blockade of properdin was protective in murine models of complement dependent disease (8) as would have been more readily predicted. It is likely that the difference between the Udea et al. model of aHUS and the $\mathrm{C} 3 \mathrm{G}$ models could be related to the way properdin interacts with $\mathrm{C} 3$ at a cell surface (plus/ minus effects on $\mathrm{FH}$ binding/FH related protein function etc.) versus in the fluid phase and how that influences C5 convertase generation. This raises questions about the use of anti-properdin drugs in certain kidney diseases or more precisely in patients with particular rare variants in $\mathrm{C} 3$ or $\mathrm{FH}$ or with particular $\mathrm{C} 3 / \mathrm{C} 5$ convertase autoantibodies (C3 \& C5 nephritic factors) that need detailed and careful examination (see Michels et al. for a comprehensive review) (17). Indeed, there may be a rational argument for use of combined therapy, with anti-properdin mAbs supplemented with use of FH module based therapies, such as TT30, mini-FH or HDM-FH $(8,18)$ but this will require considerable detailed analysis to confirm.

Unlike FH and $\mathrm{C} 3$, there does not appear to be any genetic/familial evidence for a role in properdin loss or gain-of-function in association with aHUS. Only two rare variants in properdin were reported in the 6 centres study (4) and a maximal of $5-6$ missense changes (MAF $<0.01$; normal plasma properdin expression levels) have been found associated with aHUS (Prof. Santiago Rodriquez de Cordoba-personal communication). So, a direct role of properdin variants in the development of aHUS seems 
unlikely and thus changes in other factors surrounding properdin function remain of primary focus in development of aHUS. These data may also hint that the role of properdin in TMA mediated disease is secondary to the initial complement activation and potentially links back to the prominent role of properdin in thrombo-inflammation.

\section{Conclusions}

The study by Ueda et al. has clearly demonstrated an important role for properdin in aggressive TMA mediated disease. Thus, with many questions surrounding the long-term risks of eculizumab therapy and costs/dosing regimens remaining largely unresolved, the potential to use anti-properdin or other anti-complement drugs (25) needs to be explored. Based on the result of one animal model of aHUS, anti-properdin maybe a serious option but it does come with a few caveats at the moment. The fact that $\mathrm{FH}^{\mathrm{R} / \mathrm{R}}$ mice do not just develop renal TMA but also develop systemic thrombophilia involving large blood vessels in the brain, liver, lung, spleen and kidney suggests some consideration is needed before findings in the $\mathrm{FH}^{\mathrm{R} / \mathrm{R}}$ model are extrapolated to less aggressive TMA and other complement mediated diseases. Of course before use of anti-properdin in kidney disease, clinicians would need to be certain of pathophysiological basis of the glomerular diseases that they are faced with i.e., deregulation of surface complement activation (aHUS) or deregulation of systemic complement activation (C3GN, DDD, atypical post infectious glomerulonephritis). This remains a challenge although enormous progress has been made in standardisation and provision of complement analysis, biochemical, immunological and genetic; with the message that a comprehensive diagnostic approach remains critical for these patients. Once diagnosis is clear and the appropriate controls (vaccination/prophylactic antibiotic strategies in place) use of anti-properdin therapy for treatment of severe TMA/aHUS may have a prominent place in the clinical armoury.

\section{Acknowledgements}

None.

\section{Footnote}

Conflicts of Interest: KJ Marchbank is a member of Gemini Therapeutics inc scientific advisor board and is contracted by Idorsia ltd to test agents in the C3 GOF mouse. K Smith-Jackson has no conflicts of interest to declare.

\section{References}

1. Brocklebank V, Kavanagh D. Complement C5inhibiting therapy for the thrombotic microangiopathies: accumulating evidence, but not a panacea. Clin Kidney J 2017;10:600-24.

2. Ueda Y, Miwa T, Gullipalli D, et al. Blocking Properdin Prevents Complement-Mediated Hemolytic Uremic Syndrome and Systemic Thrombophilia. J Am Soc Nephrol 2018;29:1928-37.

3. Alexion. Soliris (Eculizumab). 2011. Available online: https://www.solirisrems.com/docs/sol1135_soliris_dosing_ and_admin_brochure.pdf

4. Osborne AJ, Breno M, Borsa NG, et al. Statistical Validation of Rare Complement Variants Provides Insights into the Molecular Basis of Atypical Hemolytic Uremic Syndrome and C3 Glomerulopathy. J Immunol 2018;200:2464-78.

5. Pickering MC, de Jorge EG, Martinez-Barricarte R, et al. Spontaneous hemolytic uremic syndrome triggered by complement factor $\mathrm{H}$ lacking surface recognition domains. J Exp Med 2007;204:1249-56.

6. Ueda Y, Mohammed I, Song D, et al. Murine systemic thrombophilia and hemolytic uremic syndrome from a factor H point mutation. Blood 2017;129:1184-96.

7. Kaplan BS, Ruebner RL, Spinale JM, et al. Current treatment of atypical hemolytic uremic syndrome. Intractable Rare Dis Res 2014;3:34-45.

8. Blatt AZ, Pathan S, Ferreira VP. Properdin: a tightly regulated critical inflammatory modulator. Immunol Rev 2016;274:172-90.

9. Holers VM, Thurman JM. The alternative pathway of complement in disease: opportunities for therapeutic targeting. Mol Immunol 2004;41:147-52.

10. Morgan BP, Marchbank KJ, Longhi MP, et al. Complement: central to innate immunity and bridging to adaptive responses. Immunol Lett 2005;97:171-9.

11. Kimura Y, Miwa T, Zhou L, et al. Activator-specific requirement of properdin in the initiation and amplification of the alternative pathway complement. Blood 2008;111:732-40.

12. Barnum S, Schein T. The Complement Facts Book. Second edition. Massachusetts: Academic Press, 2018.

13. Huugen D, van Esch A, Xiao H, et al. Inhibition of complement factor $\mathrm{C} 5$ protects against anti- 
myeloperoxidase antibody-mediated glomerulonephritis in mice. Kidney Int 2007;71:646-54.

14. Bertram P, Akk AM, Zhou HF, et al. Anti-mouse properdin TSR 5/6 monoclonal antibodies block complement alternative pathway-dependent pathogenesis. Monoclon Antib Immunodiagn Immunother 2015;34:1-6.

15. Wong EK, Goodship TH, Kavanagh D. Complement therapy in atypical haemolytic uraemic syndrome (aHUS). Mol Immunol 2013;56:199-212.

16. Linton SM, Morgan BP. Properdin deficiency and meningococcal disease--identifying those most at risk. Clin Exp Immunol 1999;118:189-91.

17. Michels MA, Volokhina EB, van de Kar NC, et al. The role of properdin in complement-mediated renal diseases: a new player in complement-inhibiting therapy? Pediatr Nephrol 2018. [Epub ahead of print].

18. Yang Y, Denton H, Davies OR, et al. An Engineered Complement Factor H Construct for Treatment of C3 Glomerulopathy. J Am Soc Nephrol 2018;29:1649-61.

19. Ricklin D, Mastellos DC, Reis ES, et al. The renaissance of complement therapeutics. Nat Rev Nephrol

Cite this article as: Smith-Jackson K, Marchbank KJ. Targeting properdin in the treatment of atypical haemolytic uraemic syndrome: better than eculizumab? Ann Transl Med 2018;6(Suppl 1):S62. doi: 10.21037/atm.2018.10.35
2018;14:26-47.

20. McNamara LA, Topaz N, Wang X, et al. High Risk for Invasive Meningococcal Disease Among Patients Receiving Eculizumab (Soliris) Despite Receipt of Meningococcal Vaccine. Am J Transplant 2017;17:2481-4.

21. Smith-Jackson K, Denton H, Cook K, et al. A novel C3 gain of function mouse model of atypical haemolytic uraemic syndrome. Immunobiology 2016;221:1136.

22. Smith-Jackson K, Yang Y, Denton H, et al. Treatment of the $\mathrm{C} 3$ gain-of-function mouse model of aHUS with BB5.1, TT30 or genetic deletion of C5. Molecular Immunology 2017;89:180.

23. Ruseva MM, Vernon KA, Lesher AM, et al. Loss of properdin exacerbates $\mathrm{C} 3$ glomerulopathy resulting from factor H deficiency. J Am Soc Nephrol 2013;24:43-52.

24. Lesher AM, Zhou L, Kimura Y, et al. Combination of factor $\mathrm{H}$ mutation and properdin deficiency causes severe C3 glomerulonephritis. J Am Soc Nephrol 2013;24:53-65.

25. Harris CL. Expanding horizons in complement drug discovery: challenges and emerging strategies. Semin Immunopathol 2018;40:125-40. 